\title{
Baryon number nonconservation as Peccei-Quinn mechanism
}

\author{
Takahiro Ohata, ${ }^{1}$ Kengo Takeuchi, ${ }^{2}$ and Koji Tsumura $\odot^{2, *}$ \\ ${ }^{1}$ Department of Physics, Kyoto University, Kyoto 606-8502, Japan \\ ${ }^{2}$ Department of Physics, Kyushu University, 744 Motooka, Nishi-ku, \\ Fukuoka 819-0395, Japan
}

(Received 3 May 2021; accepted 27 July 2021; published 26 August 2021)

\begin{abstract}
Baryon number is an accidental symmetry in the standard model, while Peccei-Quinn symmetry is hypothetical symmetry which is introduced to solve the strong $C P$ problem. We study the possible connections between Peccei-Quinn symmetry and baryon number symmetry. In this framework, an axion is identified as the Nambu-Goldstone boson of baryon number violation. As a result, characteristic baryon number violating processes are predicted. We developed the general method to determine the baryon number and lepton number of new scalar in the axion model.
\end{abstract}

DOI: 10.1103/PhysRevD.104.035026

\section{INTRODUCTION}

The last undetermined parameter of the standard model for particle physics (SM) is the QCD $\theta$ parameter. The $\theta$ parameter is constrained to be extremely small by the search for the neutron electric dipole moment [1], which imply the $C P$ symmetry in the strong interaction. However, the $C P$ symmetry is broken in the electroweak sector of the SM by the Kobayashi-Maskawa mechanism. It is natural to expect the $C P$ violation in the strong interaction against nonobservation of the strong $C P$ phase $\theta$. This unnaturalness is called the strong $C P$ problem [2].

The strong $C P$ problem may be solved by the hypothetical Peccei-Quinn symmetry (PQ symmetry)[3], where the enhanced global symmetry leaves an axion as NambuGoldstone boson after the symmetry breaking. Thanks to the shift symmetry of the axion, the QCD $\theta$ parameter becomes unphysical. When the axion develops a vacuum expectation value (VEV), the vanishing $\theta$ parameter is realized as a physical quantity. Thus, the strong $C P$ problem is solved dynamically. The minimal extension of the SM to realize the PQ mechanism is known to be ruled out, where the second Higgs doublet is introduced with a global symmetry [4]. There are two major axion models. One is so-called KSVZ axion [5], where the PQ mechanism is realized outside the SM sector. An SM singlet complex scalar is coupled to the postulated heavy colored fermions. The other is DFSZ axion model [6], where an SM singlet

\footnotetext{
tsumura.koji@phys.kyushu-u.ac.jp
}

Published by the American Physical Society under the terms of the Creative Commons Attribution 4.0 International license. Further distribution of this work must maintain attribution to the author(s) and the published article's title, journal citation, and DOI. Funded by SCOAP. complex scalar is added to the original axion model [4]. Assuming the large VEV of the singlet scalar, these axion models become invisible against experimental searches.

Invisible axion models are often combined with the global symmetries, which are motivated by the other problem in the SM. Froggatt-Nielsen mechanism is a familiar mechanism of the origin of fermion mass hierarchy, where a flavor symmetry is assumed. If the flavor symmetry and the PQ symmetry are broken by the common $\mathrm{VEV}$, then these two independently inspired scenarios have the common physics scale and the characterized predictions $[7,8]$. For another example of such model, the type-I seesaw mechanism is known to generate the lepton number violating Majorana neutrino masses. If the lepton number symmetry is identified with PQ symmetry, a pseudo Nambu-Goldstone boson called Majoron [9] plays a role of axion [10,11].

The search for the violation of conservation law is believed to be a useful probe to access the physics far beyond the $\mathrm{TeV}$ scale. Although LHC has been reported null evidences of new physics below $\mathrm{TeV}$ scale, indirect searches using flavor/ number as well as $C P$ violation have been explored the high energy scale much higher than the $\mathrm{TeV}$ scale through the virtual mediator effects. Nonobservation of the nucleon decay search has been provided one of the most stringent bound on such process [12]. The searches of the lepton number violating neutrinoless double beta decay and the lepton flavor violating processes have also been known to be very sensitive probes of new physics.

In the classical level of the SM, the baryon number $\mathbf{B}$ and lepton number $\mathbf{L}$ are accidentally conserved. However, in the SM effective field theory, these symmetries are, in general, violated by the higher dimensional operators. For instance, the $\mathbf{L}$ violating operator $L L H H$ is allowed at dimension-five, while $\mathbf{B}+\mathbf{L}$ violating operators are 
allowed at dimension-six [13,14]. Depending on the operator dimensions, characteristic number violating processes are predicted [13-15]. If these number violation is identified as PQ symmetry breaking, the PQ mechanism may also be explored by the powerful number violation searches.

In this paper, we propose new axion models based on the lepton and/or baryon number conservation. These number symmetries are identified as the PQ symmetry. The strong $C P$ problem is solved by ordinary PQ mechanism, while the characteristic number violation is predicted. The method to identify the lepton number symmetry by the PQ symmetry is developed in Majoraxion models, where the lepton number symmetry and PQ symmetry is identified. We then generalized the method with the baryon number symmetry. As typical examples, we construct axion models which predict nucleon decays, $n-\bar{n}$ oscillation, and dinucleon decays.

This paper is organized as follows. In Sec. II, the Majoraxion models are reviewed and formulated by the model independent framework. A method to identify the number symmetry by PQ symmetry is developed. In Sec. III, axion models based on the baryon (and lepton) number violations are proposed with typical baryon number violating experimental signatures. Conclusion and discussion are given in Sec. IV.

\section{ANATOMY OF MAJORAXION MODELS}

In this section, we review on known Majoraxion models, and then the idea is reformulated in the model independent framework using the higher order operators.

The idea of the Majoraxion is to unify the axion and the Majoron, where the former is a pseudo-Nambu-Goldstone boson (pNGB) induced by the PQ symmetry breaking while the latter is one from the breaking of the lepton number symmetry. The first model [10] of this category is based on the connection between the type-I seesaw model and the KSVZ axion model. In the type-I seesaw model [16], the SM singlet right-handed neutrinos $N_{R}$ are postulated to generate the observed neutrino mass and mixing. In this model, the lepton number conservation is broken explicitly by Majorana mass for right-handed neutrinos. The lepton number symmetry can be restored by introducing the SM singlet complex scalar $S$ as

$$
\mathcal{L}_{N}=\left(-y_{N} \bar{L} \tilde{H} N_{R}+\text { H.c. }\right)-\frac{1}{2} h_{N} S^{*} \bar{N}_{R}^{C} N_{R},
$$

where $L$ and $H$ are the lepton and Higgs doublet in the $\mathrm{SM}$, respectively. We also note $\tilde{H}=i \tau_{2} H^{*}$ and $\psi^{C}=C \bar{\psi}^{t}$. Through Yukawa interaction with lepton numberless coupling constant, the lepton numbers $\mathbf{L}$ of $N_{R}$ and $S$ are uniquely determined, i.e., $\mathbf{L}\left(N_{R}\right)=+1$ and $\mathbf{L}(S)=+2$. In Shin's model [10], the common complex scalar $S$ is used to break the PQ symmetry of KSVZ axion model, ${ }^{1}$

$$
\mathcal{L}_{\Psi}=-y_{\Psi} S^{*} \bar{\Psi}_{L} \Psi_{R}+\text { H.c. }
$$

where $\Psi_{L}$ and $\Psi_{R}$ are so-called KSVZ quarks. It is now clear that since $\mathbf{L}\left(\bar{\Psi}_{L} \Psi_{R}\right) \neq 0$, the lepton number symmetry plays a role of PQ symmetry in this setup. Thus, the Majoron is identified as the axion. As the simplest choice, $\Psi_{L}$ and $\Psi_{R}$ are assumed to transform as a fundamental representation of QCD and have single flavor in order to easily avoid the domain wall problem [19]. If we write the KSVZ Yukawa interaction with $S$ as

$$
\mathcal{L}_{\Psi}^{\prime}=-y_{\Psi} S \bar{\Psi}_{L} \Psi_{R}+\text { H.c. }
$$

a different lepton numbers assignment is possible.

Let us reformulate the idea of Majoraxion in a model independent way. At renormalizable level, the (global) lepton number symmetry is an accidental symmetry of the SM. On the other hand, the lepton number is in general broken by higher dimensional operators constructed by the SM fields. The most popular lepton number violating operator is socalled Weinberg operator with mass dimension five [13],

$$
\hat{\mathcal{O}}_{5}=L L H H .
$$

This operator breaks lepton number by two units, $\Delta \mathbf{L}=2$. What was done in the Majoraxion model is to restore the lepton number symmetry by introducing the complex scalar $S$, i.e.,

$$
\mathcal{O}_{6}=S^{*} \hat{\mathcal{O}}_{5}=S^{*} L L H H,
$$

where the lepton number of $S$ is fixed to be $\mathbf{L}(S)=+2$. Now, $S$ has a well-defined lepton number by Eq. (5), which is transmitted to $\Psi_{L}$ and/or $\Psi_{R}$ through the KSVZ Yukawa interaction in Eq. (3). This prescription is further developed with the baryon number symmetry in the next section. When $S$ acquires the VEV with this lepton number conserving operator, the Majoron appears as an axion. If we begin with a seesaw model which has lepton number violating dimensionful parameter, the Majoraxion model is derived by replacing this operator with the complex scalar $S$. That was done in the above example.

This prescription is applied for the type-II seesaw model [20], where a complex triplet scalar $\Delta$ with $Y=1$ is introduced. ${ }^{2}$ In the type-II seesaw model, a dimensionful

${ }^{1}$ The model can also be combined with DFSZ axion model $[17,18]$. As discussed in Ref. [18], $U(1)_{\mathrm{PQ}}$ is entangled with $U(1)_{\mathbf{B}}, U(1)_{\mathbf{L}}$ and $U(1)_{Y}$ in the DFSZ model due to the ambiguity of PQ charges of SM fermions. On the other hand, PQ symmetry is broken only by SM singlet scalar in the KSVZ model resulting no entanglement. Thus, PQ symmetry can be identified as a part of $U(1)_{\mathbf{B}} \times U(1)_{\mathbf{L}}$ symmetry.

${ }^{2}$ Our hypercharge convention is $Q_{\mathrm{EM}}=T_{3}+Y$, where the electric charge $Q_{\mathrm{EM}}$ and the third component of weak isospin $T_{3}$. 

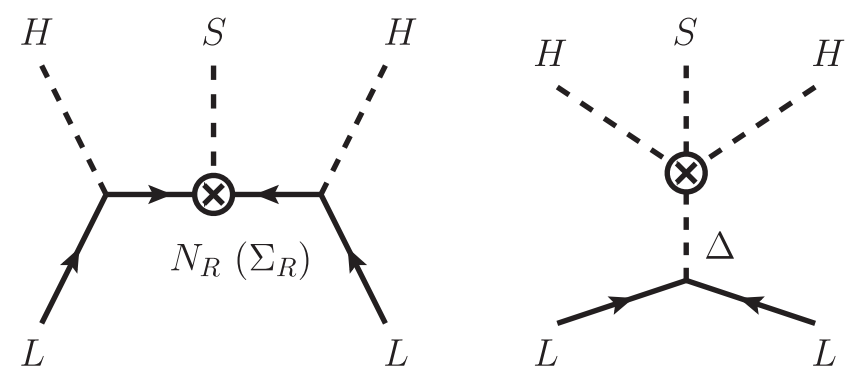

FIG. 1. Diagrams for tree-level Majoraxion models.

parameter with lepton number violation is $\mu$ in the scalar potential, $\mathcal{V} \sim \mu H^{*} \Delta H^{*}$, where the lepton number of $\Delta$ is rigorously fixed by the Yukawa interaction $y_{\Delta} L \Delta L$. Therefore, by promoting $\mu$ as $S$ we obtain the Majoraxion extension of the type-II seesaw model [21]. The Majoraxion extension [22] of type-III seesaw model [23] is nothing different from the type-I seesaw model, where the right-handed neutrino is simply replaced by the triplet fermion $\Sigma_{R}$ with $Y=0$. In Fig. 1, the diagrams for $\mathcal{O}_{6}$ are given in type-I (left) and in type-II (right) Majoraxion models. The case for type-III seesaw extension is also shown in the left panel. If we truncate the external $S$ line in these diagram, we obtain the diagrams for the neutrino mass generation of lepton number violating dimension five operator $\hat{\mathcal{O}}_{5}$ in ordinary seesaw mechanism.

The higher dimensional operator $\mathcal{O}_{6}$ is not necessarily decomposed by tree diagrams. The above mentioned type-I -II -III seesaw models are based on the tree level decomposition [24] of the prototype operator of $\hat{O}_{5}$. The loop level classification of $\hat{O}_{5}$ is called radiative seesaw model, where neutrino masses are generated by quantum corrections. The variants of radiative seesaw model have been studied very extensively (For a comprehensive review of radiative seesaw models, see [25]). There must be a loop level ultraviolet (UV) completion of $\mathcal{O}_{6}$, that is radiative Majoraxion model $[26,27]$. The extension of these radiative seesaw model to the Majoraxion model is straightforward. In Fig. 2, the diagrams for the radiative Majoraxion extensions of Zee model (left), Zee-Babu model (center) and scotogenic model (right). In ordinary Zee model [28], a pair of singly charged scalar $k^{ \pm}$and an extra Higgs doublet $H^{\prime}$ are introduced in order to form the lepton number violating connection $\mu_{\mathrm{Z}} k^{+} H^{*} H^{*}$ with dimensionful coupling $\mu_{\mathrm{Z}}$. The substitution of $\mu_{\mathrm{Z}}$ by $S$ again identifies the lepton number symmetry as PQ symmetry [29]. In ordinary Zee-Babu model [30], a pair of doubly charged scalar $k^{ \pm \pm}$ is added instead $H^{\prime}$, then the lepton number violating dimensionful coupling $\mu_{\mathrm{ZB}}$ is allowed as $\mu_{\mathrm{ZB}} k^{+} k^{+} k^{--}$. By substituting $\mu_{\mathrm{Z}}$ by $S$, a Majoraxion extension of Zee-Babu model is realized [21]. In the scotogenic model [31], righthanded neutrinos and the so-called inert doublet are assumed to be odd under the ad hoc $Z_{2}$ symmetry in order to make the dark matter stable. In the Majoraxion extension of the scotogenic model, the dimensionful parameter is provided by the Majorana mass of right-handed neutrinos as in type-I seesaw model. The stability of the dark matter is automatically guaranteed by the residual $Z_{2}$ symmetry (lepton parity [32,33], which is lead by the breakdown of the global lepton number symmetry a la Krauss-Wilczek mechanism [34].

\section{B AND L VIOLATION AS PQ MECHANISM}

In this section, we focus on the lepton number and baryon number violating operators with mass dimensions more than $d=5$. At $d=6, \mathbf{B}-\mathbf{L}$ conserving operators of $q q q \ell$ are allowed, where $q$ and $\ell$ are general quark and lepton fields. The $d=7$ operators of $q q q \bar{\ell} \varphi$ hold $\mathbf{B}+\mathbf{L}$ but violate $\mathbf{B}-\mathbf{L}$, where $\varphi$ denotes a SM boson field or space-time derivative. In this paper, we assume $\varphi$ to be the Higgs field $H$ or its charge conjugation. For the characterization of $d>7$ baryon/lepton number violating operators, see for example [15].

\section{A. Axion models based on $\Delta(B+L)=2$ symmetry breaking}

The $d=6$ operators of qqq $\ell$ type break both the baryon number and lepton number by one unit as $\Delta \mathbf{B}=\Delta \mathbf{L}=1$. These are given by

$$
\hat{\mathcal{O}}_{6}=\left\{u_{R} u_{R} d_{R} e_{R}, u_{R} d_{R} Q L, u_{R} Q Q e_{R}, Q Q Q L\right\},
$$
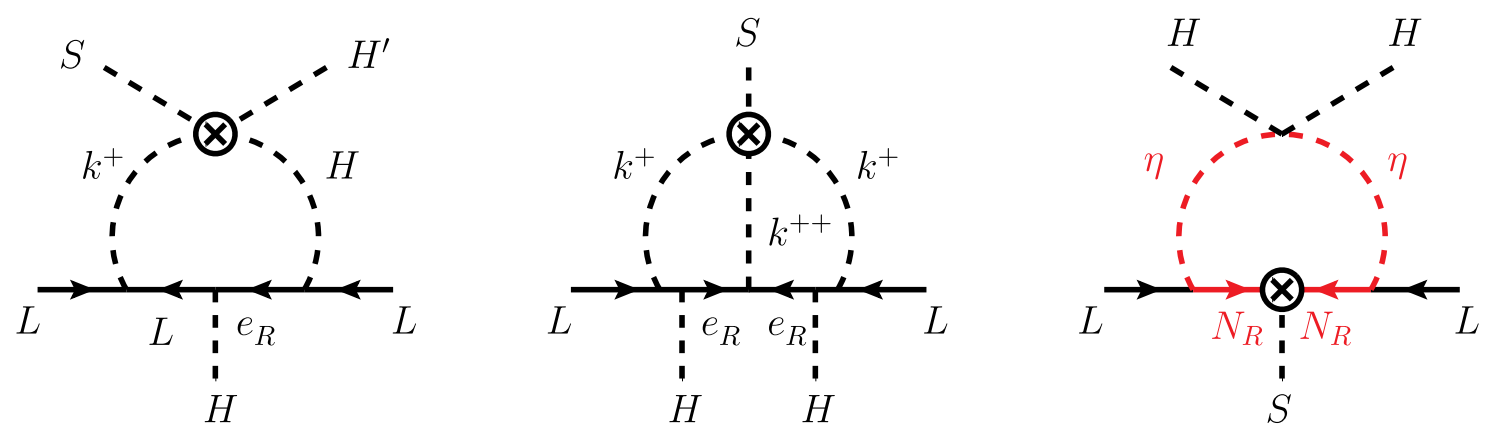

FIG. 2. Diagrams for loop-level Majoraxion models. 
where SM right-handed quark singlets $u_{R}, d_{R}$ and lefthanded quark doublet $Q$, respectively. All these operators hold global $\mathbf{B}-\mathbf{L}$ symmetry, while the $\mathbf{B}+\mathbf{L}$ symmetry is explicitly broken. According to the prescription discussed in the previous section, $\mathbf{B}+\mathbf{L}$ symmetry may be restored by introducing a complex scalar $S$ as

$$
\mathcal{O}_{7}=S^{*} \hat{\mathcal{O}}_{6}
$$

Note that the baryon and lepton numbers of $S$ are determined as $\mathbf{B}(S)=\mathbf{L}(S)=+1$ through this operator. In general, the $\mathbf{B}+\mathbf{L}$ conserving operator may be higher order as $\mathcal{O}_{8}=\left(S^{*}\right)^{2} \hat{\mathcal{O}}_{6}, \cdots$, instead. If we commonly use $S^{*}$ or $S$ for the KSVZ Yukawa interaction as in Eq. (2) or Eq. (3), pNGB caused by $\mathbf{B}+\mathbf{L}$ symmetry breaking is identified as an axion. At this point, how can we assign $\mathbf{B}$ and $\mathbf{L}$ for $\Psi_{L}$ and $\Psi_{R}$ ?

The $\mathbf{B}$ and $\mathbf{L}$ charges for $\Psi_{L}$ and $\Psi_{R}$ can be determined by introducing the one of the following mass mixing operators,

$$
\mathcal{O}_{\text {mix }}^{\prime}=\left\{\bar{Q} \tilde{H} \Psi_{R}^{U}, \overline{\Psi_{L}^{U}} u_{R}, \bar{Q} H \Psi_{R}^{D}, \overline{\Psi_{L}^{D}} d_{R}\right\}
$$

The hypercharges of $\Psi_{L}^{q}$ and $\Psi_{R}^{q}$ are chosen to be $+2 / 3$ (for $q=U$ ) or $-1 / 3$ (for $q=D$ ). These operators are obtained by replacing a SM quark singlet $q_{R}\left(=u_{R}, d_{R}\right)$ by $\Psi_{R}^{q}$ or $\bar{Q} \tilde{H}(\bar{Q} H)$ by $\overline{\Psi_{L}^{U}}\left(\bar{\Psi}_{L}^{D}\right)$ in the quark Yukawa interactions, i.e., $y_{U} \bar{Q} \tilde{H} u_{R}$ and $y_{D} \bar{Q} H d_{R}$. This is usually done in the KSVZ model in order to allow the decay of KSVZ quark into the SM particles. Through these connections, we find $\mathbf{B}\left(\Psi_{L}^{q}\right)=\mathbf{B}\left(\Psi_{R}^{q}\right)=+1 / 3$ and $\mathbf{L}\left(\Psi_{L}^{q}\right)=\mathbf{L}\left(\Psi_{R}^{q}\right)=0$. This is not the case for our purpose. In order to identify the pNGB as an axion, $\Psi_{L}$ and $\Psi_{R}$ must have different $\mathbf{B}$ and $\mathbf{L}$ charges. Therefore, we determine $\mathbf{B}$ and $\mathbf{L}$ only for
$\Psi_{L}^{q}\left(\Psi_{R}^{q}\right)$ by this procedure, and the other link for $\Psi_{R}^{q}\left(\Psi_{L}^{q}\right)$ is taken from the KSVZ Yukawa interactions through Eq. (7). As a concrete example, we assume the operators of $\overline{\Psi_{L}^{D}} d_{R}$ and $S^{*} Q Q Q L$ in our effective Lagrangian. At this point, $\mathbf{B}$ and $\mathbf{L}$ for $\Psi_{L}^{D}$ and $S$ are uniquely fixed, while that for $\Psi_{R}^{D}$ is ambiguous. Once the KSVZ Yukawa interaction $S \overline{\Psi_{L}^{D}} \Psi_{R}^{D}$ is turned on, $\mathbf{B}$ and $\mathbf{L}$ for $\Psi_{R}^{D}$ are uniquely determined.

The $\mathbf{B}+\mathbf{L}$ conserving operator $\mathcal{O}_{7}$ can be systematically constructed from the following $\mathbf{B}$ and $\mathbf{L}$ conserving operators,

$$
\begin{aligned}
\mathcal{O}_{6}^{\prime}= & \left\{\Psi_{R}^{U} u_{R} d_{R} e_{R}, u_{R} u_{R} \Psi_{R}^{D} e_{R},\right. \\
& \left.\Psi_{R}^{U} d_{R} Q L, u_{R} \Psi_{R}^{D} Q L, \Psi_{R}^{U} Q Q e_{R}\right\},
\end{aligned}
$$

where one of a SM quark singlet $q_{R}$ is substituted by $\Psi_{R}^{q}$ in $\hat{\mathcal{O}}_{6}$. Similarly to the method developed with $\mathcal{O}_{\text {mix }}^{\prime}, \mathcal{O}_{6}^{\prime}$ determines the baryon (lepton) number of KSVZ quarks as $\mathbf{B}\left(\Psi_{R}^{q}\right)=-2 / 3$ and $\mathbf{L}\left(\Psi_{R}^{q}\right)=-1$. If we take $\Psi_{L}^{q}$ from $\mathcal{O}_{\text {mix }}^{\prime}$ and $\Psi_{R}^{q}$ from $\mathcal{O}_{6}^{\prime}$, the KSVZ Yukawa interaction in Eq. (3) induces $\mathcal{O}_{7}$. The higher order operator $\mathcal{O}_{8}$ can also be derived by considering the operator $\mathcal{O}_{6}^{\prime \prime}=d_{R} \Psi_{R}^{U} \Psi_{R}^{U} e_{R}$, where $\mathbf{B}\left(\Psi_{R}^{U}\right)=-1 / 6$ and $\mathbf{L}\left(\Psi_{R}^{U}\right)=-1 / 2$ correspond to $\mathbf{B}(S)=\mathbf{L}(S)=+1 / 2$.

Let us show a UV complete example by introducing a singlet scalar $\xi$ with $Y=-1 / 3$. The leptoquark $\xi$ transforms as a fundamental representation of $\mathrm{QCD}$, and the baryon and lepton numbers are assigned to $\mathbf{B}(\xi)=-1 / 3$ and $\mathbf{L}(\xi)=+1$. The baryon and lepton number conservation are assumed as PQ symmetry. Then, the dimension-six interaction of $\Psi_{R}^{U} d_{R} Q L$ is decomposed to the renormalizable interactions. The relevant Lagrangian is given by

$$
\begin{aligned}
\mathcal{L}= & -y_{\Psi} S \Psi_{L}^{\bar{U} a} \Psi_{R}^{U a}-\mu_{U}^{i} \Psi_{L}^{\bar{U} a} u_{i R}^{a}-y_{\Psi D}^{i} \epsilon_{a b c} \xi^{a} \overline{\left(\Psi_{R}^{U b}\right)^{C}} d_{i R}^{c} \\
& -\left[+y_{Q L}^{i j} \overline{\left(Q_{i}^{a}\right)^{C}}\left(i \sigma_{2}\right) L_{j}+y_{U E}^{i j} \overline{\left(u_{i R}^{a}\right)^{C}} e_{R j}\right]\left(\xi^{a}\right)^{*}+\text { H.c. }
\end{aligned}
$$

We here show the color indices $(a, b, c=1,2,3)$ and the flavor indices $(i, j=1,2,3)$ explicitly, and $\epsilon_{a b c}$ is the Levi-Civita tensor. The Lagrangian is assumed to hold global $\mathbf{B}+\mathbf{L}$ symmetry as PQ symmetry. The operators of $Q Q \xi$ and $u_{R} d_{R} \xi$, which lead dangerous dimension-six nucleon decays, are forbidden by $\mathbf{B}+\mathbf{L}$ symmetry unlike ordinary leptoquark models. Note that $\xi$ has definite $\mathbf{B}$ and $\mathbf{L}$ by construction, no diquark interaction is allowed. On the other hand, the model predicts nucleon decays via $\mathcal{O}_{7}$. The corresponding Feynman diagram is depicted in the left of Fig. 3. The low energy effective Lagrangian for the nucleon decay is derived as

$$
\mathcal{L}_{\text {eff }}^{\Delta(\mathbf{B}+\mathbf{L})=2}=\epsilon_{a b c} \overline{\left(u_{i R}^{a}\right)^{C}} d_{j R}^{b}\left[+C_{U D Q L}^{i j k l}\left(\overline{\left(u_{k L}^{c}\right)^{C}} e_{l L}-\overline{\left(d_{k L}^{d}\right)^{C}} \nu_{l L}\right)+C_{U D U E}^{i j k l} \overline{\left(u_{k R}^{c}\right)^{C}} e_{l R}\right]+\text { H.c. }
$$

Here and hereafter, we omit the flavor indices. The Wilson coefficients are given by

$$
C_{U D Q L}^{i j k l}=-\frac{\mu_{U}^{i} y_{\Psi D}^{j} y_{Q L}^{k l}}{M_{\Psi} M_{\xi}^{2}}, \quad C_{U D U E}^{i j k l}=-\frac{\mu_{U}^{i} y_{\Psi D}^{j} y_{u e}^{k l}}{M_{\Psi} M_{\xi}^{2}}
$$


where mass of leptoquark is $M_{\xi}$, and that of $\mathrm{KSVZ}$ quark is $M_{\Psi}=y_{\Psi}\langle S\rangle .{ }^{3}$ Using these effective interactions, the proton decay rates are

$$
\begin{gathered}
\Gamma_{p \rightarrow \pi^{0} e_{i}^{+}}=\frac{m_{p}}{32 \pi}\left(1-\frac{m_{\pi}^{2}}{m_{p}^{2}}\right)^{2}\left(\left|C_{U D Q L}^{111 i}\left\langle\pi^{0}\left|(u d)_{R} u_{L}\right| p\right\rangle_{i}\right|^{2}+\left|C_{U D U E}^{111 i}\left\langle\pi^{0}\left|(u d)_{L} u_{L}\right| p\right\rangle_{i}\right|^{2}\right), \\
\Gamma_{p \rightarrow \pi^{+} \bar{\nu}_{i}}=\frac{m_{p}}{32 \pi}\left(1-\frac{m_{\pi}^{2}}{m_{p}^{2}}\right)^{2}\left|C_{U D Q L}^{111 i}\left\langle\pi^{+}\left|(d u)_{R} d_{L}\right| p\right\rangle\right|^{2}, \\
\Gamma_{p \rightarrow K^{0} e_{i}^{+}}=\frac{m_{p}}{32 \pi}\left(1-\frac{m_{K}^{2}}{m_{p}^{2}}\right)^{2}\left(\left|C_{U D Q L}^{121 i}\left\langle K^{0}\left|(u s)_{R} u_{L}\right| p\right\rangle_{i}\right|^{2}+\left|C_{U D U E}^{121 i}\left\langle K^{0}\left|(u s)_{L} u_{L}\right| p\right\rangle_{i}\right|^{2}\right) \\
\Gamma_{p \rightarrow K^{+} \bar{\nu}_{i}}=\frac{m_{p}}{32 \pi}\left(1-\frac{m_{K}^{2}}{m_{p}^{2}}\right)^{2}\left|C_{U D Q L}^{121 i}\left\langle K^{+}\left|(u s)_{R} d_{L}\right| p\right\rangle+C_{U D Q L}^{112 i}\left\langle K^{+}\left|(u d)_{R} s_{L}\right| p\right\rangle\right|^{2}, \\
\Gamma_{p \rightarrow \eta^{0} e_{i}^{+}}=\frac{m_{p}}{32 \pi}\left(1-\frac{m_{\eta}^{2}}{m_{p}^{2}}\right)^{2}\left(\left|C_{U D Q L}^{111 i}\left\langle\eta^{0}\left|(u d)_{R} u_{L}\right| p\right\rangle_{i}\right|^{2}+\left|C_{U D U E}^{111 i}\left\langle\eta^{0}\left|(u d)_{L} u_{L}\right| p\right\rangle_{i}\right|^{2}\right)
\end{gathered}
$$

where the nucleon matrix elements are taken from the lattice simulation result [35]. For simplicity, we assume $y_{Q L}^{i j}=y_{u e}^{i j}=y_{q \ell}, y_{\Psi D}^{i}=y_{\Psi D}$ and $\mu_{U}^{i}=\mu_{U}$ in numerical estimates. The proton (partial) lifetime is evaluated as functions of model parameters as

$$
\begin{aligned}
& \tau_{p \rightarrow \pi^{0} e^{+}} \simeq\left(2.4 \times 10^{34} \mathrm{yrs}\right) \times\left(\frac{0.2}{\mu_{U} / M_{\Psi}}\right)^{2}\left(\frac{1}{\left|y_{\Psi D}\right|}\right)^{2}\left(\frac{1}{\left|y_{q \ell}\right|}\right)^{2}\left(\frac{M_{\xi}}{2.0 \times 10^{15} \mathrm{GeV}}\right)^{4}, \\
& \tau_{p \rightarrow \pi^{0} \mu^{+}} \simeq\left(1.6 \times 10^{34} \mathrm{yrs}\right) \times\left(\frac{0.2}{\mu_{U} / M_{\Psi}}\right)^{2}\left(\frac{1}{\left|y_{\Psi D}\right|}\right)^{2}\left(\frac{1}{\left|y_{q \ell}\right|}\right)^{2}\left(\frac{M_{\xi}}{1.7 \times 10^{15} \mathrm{GeV}}\right)^{4}, \\
& \tau_{p \rightarrow \pi^{+} \bar{\nu}_{i}} \simeq\left(3.9 \times 10^{32} \mathrm{yrs}\right) \times\left(\frac{0.2}{\mu_{U} / M_{\Psi}}\right)^{2}\left(\frac{1}{\left|y_{\Psi D}\right|}\right)^{2}\left(\frac{1}{\left|y_{q \ell}\right|}\right)^{2}\left(\frac{M_{\xi}}{6.9 \times 10^{14} \mathrm{GeV}}\right)^{4}, \\
& \tau_{p \rightarrow K^{0} e^{+}} \simeq\left(1.0 \times 10^{33} \mathrm{yrs}\right) \times\left(\frac{0.2}{\mu_{U} / M_{\Psi}}\right)^{2}\left(\frac{1}{\left|y_{\Psi D}\right|}\right)^{2}\left(\frac{1}{\left|y_{q \ell}\right|}\right)^{2}\left(\frac{M_{\xi}}{6.0 \times 10^{14} \mathrm{GeV}}\right)^{4}, \\
& \tau_{p \rightarrow K^{0} \mu^{+}} \simeq\left(1.6 \times 10^{33} \mathrm{yrs}\right) \times\left(\frac{0.2}{\mu_{U} / M_{\Psi}}\right)^{2}\left(\frac{1}{\left|y_{\Psi D}\right|}\right)^{2}\left(\frac{1}{\left|y_{q \ell}\right|}\right)^{2}\left(\frac{M_{\xi}}{6.7 \times 10^{14} \mathrm{GeV}}\right)^{4}, \\
& \tau_{p \rightarrow K^{+} \bar{\nu}_{i}} \simeq\left(6.6 \times 10^{33} \mathrm{yrs}\right) \times\left(\frac{0.2}{\mu_{U} / M_{\Psi}}\right)^{2}\left(\frac{1}{\left|y_{\Psi D}\right|}\right)^{2}\left(\frac{1}{\left|y_{q \ell}\right|}\right)^{2}\left(\frac{M_{\xi}}{1.2 \times 10^{15} \mathrm{GeV}}\right)^{4}, \\
& \tau_{p \rightarrow \eta^{0} e^{+}} \simeq\left(1.0 \times 10^{34} \mathrm{yrs}\right) \times\left(\frac{0.2}{\mu_{U} / M_{\Psi}}\right)^{2}\left(\frac{1}{\left|y_{\Psi D}\right|}\right)^{2}\left(\frac{1}{\left|y_{q \ell}\right|}\right)^{2}\left(\frac{M_{\xi}}{1.0 \times 10^{15} \mathrm{GeV}}\right)^{4}, \\
& \tau_{p \rightarrow \eta^{0} \mu^{+}} \simeq\left(4.7 \times 10^{33} \mathrm{yrs}\right) \times\left(\frac{0.2}{\mu_{U} / M_{\Psi}}\right)^{2}\left(\frac{1}{\left|y_{\Psi D}\right|}\right)^{2}\left(\frac{1}{\left|y_{q \ell}\right|}\right)^{2}\left(\frac{M_{\xi}}{8.1 \times 10^{14} \mathrm{GeV}}\right)^{4},
\end{aligned}
$$

\footnotetext{
${ }^{3}$ The typical mass scale of KSVZ quark can be as large as $\langle S\rangle$, while a TeV scale mass is possible by assuming the small Yukawa coupling constant $y_{\Psi} \ll 1$.
} 


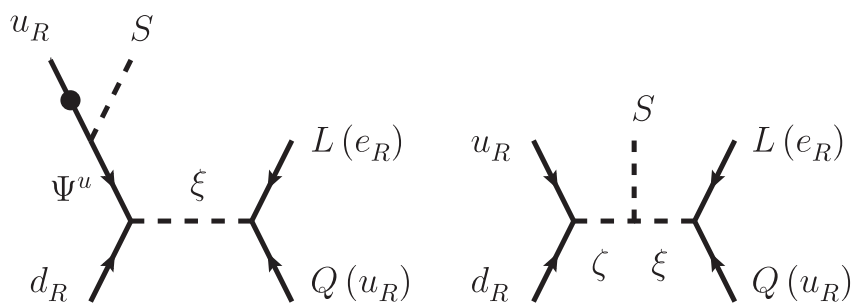

FIG. 3. Diagrams for $\mathcal{O}_{7}$ in a UV complete model.

where $\tau_{p \rightarrow M \ell_{i}}=\Gamma_{p \rightarrow M \ell_{i}}^{-1}, \mu_{U} / M_{\Psi}$ characterizes the mixing between the SM and KSVZ quark. Among the current experimental bounds [36-42] on the proton decay modes, we find that $p \rightarrow \pi^{0} e^{+}$mode gives the strongest constraint on this model. In the above formula, we normalize the partial lifetime of the proton by their current lower bound, e.g., the lower bound for $p \rightarrow \pi^{0} e^{+}$mode is $\tau_{p \rightarrow \pi^{0} e^{+}}<$ $2.4 \times 10^{34} \mathrm{yrs}$ [36]. Among all the proton decay constraints, $p \rightarrow \pi^{0} e^{+}$gives a strongest one. If we assume the order one Yukawa couplings $\left|y_{\Psi_{D}}\right| \sim\left|y_{q \ell}\right| \sim 1$ and the order one mixing $\mu_{U} / M_{\Psi} \sim 0.2$, the proton decay has already given a very stringent constraint on the new physics scale $M_{\xi}$ as $M_{\xi} \gtrsim 10^{15} \mathrm{GeV}$, which is much larger than typical PQ scale of $10^{12-14} \mathrm{GeV}$. We note that the scale of direct search of new colored particles at hadron colliders is only a few $\mathrm{TeV}$, and those of indirect searches through the flavor changing observables are about $100-1000 \mathrm{TeV}$ by assuming the order one coupling constants. Therefore, the proton decay search is the most promising way to explore this model.

Note that the other type of UV completion is also possible with more particles. For instance, if we introduce a QCD antifundamental scalar singlet diquark $\zeta$ with $Y=$ $+1 / 3$ together with $\xi$, then the new Yukawa interaction $u_{R} d_{R} \zeta^{*}$ and the source of the mass mixing between the diquark and the leptoquark, $\zeta^{*} \xi^{*} S$, are allowed. The corresponding Feynman diagram for this UV completion is shown in the right of Fig. 3 as an example.

We may add right-handed neutrinos without imposing the lepton number symmetry in these setup. Since the Majorana mass term for right-handed neutrinos breaks the continuous lepton number symmetry explicitly, the baryon number symmetry is solely identified as PQ symmetry in this case. Thus, the "Sakharaxion" scenario is realized in a minimal way, where the Sakharon [43], pNGB from the spontaneous baryon number violation, is identified as an axion.

Finally, we comment on the axion physics of the models. The model predictions for the axions are not changed from the minimal KSVZ model. This feature is common to the Majoraxion model. The constraints on the axion decay constant, e.g., from the duration time of neutrinos from the supernova SN 1987A [44], are applicable without modification. The possible axion dark matter scenario [45] can also be combined. Therefore, the model is examined only through the baryon number violation or the effect of new particles.

\section{B. Axion models based on $\Delta(B-L)=2$ symmetry breaking}

The $d=7$ operators of $q q q \bar{\ell} \varphi$ violate the baryon and lepton number as $\Delta \mathbf{B}=-\Delta \mathbf{L}=1$. which are given by

$$
\begin{gathered}
\hat{\mathcal{O}}_{7}=\left\{u_{R} d_{R} d_{R} \bar{L} H^{*}, d_{R} d_{R} d_{R} \bar{L} H,\right. \\
\left.d_{R} d_{R} Q \bar{e}_{R} H^{*}, d_{R} Q Q \bar{L} H^{*}\right\} .
\end{gathered}
$$

The global $\mathbf{B}+\mathbf{L}$ symmetry is kept, while the $\mathbf{B}-\mathbf{L}$ symmetry is not. The $\mathbf{B}-\mathbf{L}$ symmetry may be restored with a complex scalar $S$ as

$$
\mathcal{O}_{8}=S^{*} \hat{\mathcal{O}}_{7}
$$

The baryon and lepton numbers of $S$ are fixed to be $\mathbf{B}(S)=-\mathbf{L}(S)=+1$.

As discussed in the previous subsection, the $\mathbf{B}$ and $\mathbf{L}$ charges for $\Psi_{L}\left(\Psi_{R}\right)$ can be fixed by $\mathcal{O}_{\text {mix }}^{\prime}$ On the other hand, those for $\Psi_{R}\left(\Psi_{L}\right)$ is determined by the following $\mathbf{B}$ and $\mathbf{L}$ conserving operators,

$$
\begin{aligned}
\mathcal{O}_{7}^{\prime}= & \left\{\Psi_{R}^{U} d_{R} d_{R} \bar{L} H^{*}, u_{R} \Psi_{R}^{D} d_{R} \bar{L} H^{*}, \Psi_{R}^{D} d_{R} d_{R} \bar{L} H,\right. \\
& \left.\Psi_{R}^{D} d_{R} Q \bar{e}_{R} H^{*}, \Psi_{R}^{D} Q Q \bar{L} H^{*}\right\},
\end{aligned}
$$

where the SM singlet quarks are substituted by $\Psi_{R}$, and

$$
\mathcal{O}_{6}^{\prime}=\left\{d_{R} d_{R} \Psi_{L}^{D} \bar{e}_{R}, d_{R} \Psi_{L}^{D} Q \bar{L}\right\}
$$

where $Q H^{*}$ is replaced by $\Psi_{L}^{D}$ in Eq. (26). Thus, we obtain $\mathbf{B}\left(\Psi_{L / R}^{q}\right)=-2 / 3$ and $\mathbf{L}\left(\Psi_{L / R}^{q}\right)=+1$ from these connections. The extensions to higher order operators are straightforward.

As an example, we give a UV completion of this type of models by introducing a doublet scalar $\Xi$ with $Y=-2 / 3$. The $\Xi$ transforms as a fundamental representation of QCD, and the baryon and lepton numbers are assigned to $\mathbf{B}(\Xi)=$ $+2 / 3$ and $\mathbf{L}(\Xi)=-1$. The operator of $d_{R} \Psi_{L}^{D} Q \bar{L}$ is decomposed by the following renormalizable interactions

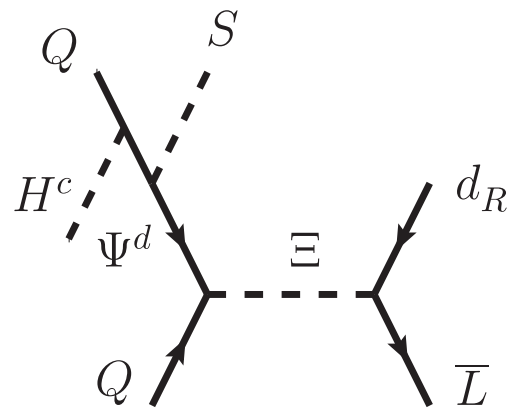

FIG. 4. Diagrams for $\mathcal{O}_{8}$ in a UV complete model. 


$$
\mathcal{L}=-y_{\Psi} S^{*} \Psi_{L}^{\bar{D} a} \Psi_{R}^{D a}-y_{D}^{i} \bar{Q}_{i}^{a} H \Psi_{R}^{D a}-y_{\Psi Q}^{i} \epsilon_{a b c} \Xi^{a} \overline{\left(\Psi_{L}^{D b}\right)^{C}} Q_{i}^{c}-y_{\bar{L} D}^{i j}\left(\Xi^{a}\right)^{*} \bar{L}_{i} d_{j R}^{a}+\text { H.c. }
$$

The global $\mathbf{B}-\mathbf{L}$ symmetry is imposed as PQ symmetry. This model predicts $\mathbf{B}+\mathbf{L}$ conserving nucleon decays via $\mathcal{O}_{8}$ as shown in Fig. 4. The low energy effective Lagrangian for the nucleon decay is derived as

$$
\mathcal{L}_{\text {eff }}^{\Delta(\mathbf{B}-\mathbf{L})=2}=C_{D Q \bar{L} D}^{i j k l} \epsilon_{a b c} \overline{\left(d_{i L}^{a}\right)^{C}}\left(u_{j L}^{b} \nu_{k L}^{-}+d_{j L}^{b} e_{k L}^{-}\right) d_{l R}^{c}+\text { H.c. }
$$

with

$$
C_{D Q \bar{L} D}^{i j k l}=-\frac{y_{D}^{i \prime *} y_{\Psi Q}^{j} y_{\bar{L} D}^{k l}}{M_{\Xi}^{2} M_{\Psi}} \frac{v_{\mathrm{EW}}}{\sqrt{2}} .
$$

where $M_{\Xi}$ denotes the mass of $\Xi$, and $v_{\mathrm{EW}}$ is the Higgs VEV of electroweak symmetry breaking. In this model, no charged lepton mode of proton decay is induced. Only neutrino modes are available

$$
\begin{gathered}
\Gamma_{p \rightarrow \pi^{+} \nu_{i}}=\frac{m_{p}}{32 \pi}\left(1-\frac{m_{\pi}^{2}}{m_{p}^{2}}\right)^{2}\left|-C_{D Q \bar{L} D}^{11 i 1}\left\langle\pi^{+}\left|(u d)_{R} d_{L}\right| p\right\rangle\right|^{2}, \\
\Gamma_{p \rightarrow K^{+} \nu_{i}}=\frac{m_{p}}{32 \pi}\left(1-\frac{m_{K}^{2}}{m_{p}^{2}}\right)^{2}\left|-C_{D Q \bar{L} D}^{21 i 1}\left\langle K^{+}\left|(u s)_{R} d_{L}\right| p\right\rangle-C_{D Q \bar{L} D}^{11 i 2}\left\langle K^{+}\left|(u d)_{R} s_{L}\right| p\right\rangle\right|^{2},
\end{gathered}
$$

where the nucleon matrix elements are determined by the lattice calculation [35]. We assume $y_{\bar{L} D}^{i j}=y_{\bar{L} D}, y_{D}^{i \prime}=y_{D}^{\prime}$, $y_{\Psi Q}^{i}=y_{\Psi Q}$ in the following numerical estimation for simplicity. The partial lifetimes calculated from inverse partial widths are

$$
\begin{aligned}
& \tau_{p \rightarrow \pi^{+} \nu_{i}} \simeq\left(3.9 \times 10^{32} \mathrm{yrs}\right) \times\left(\frac{10^{-9}}{\frac{y_{D}^{\prime} v_{\mathrm{EW}}}{\sqrt{2}} / M_{\Psi}}\right)^{2}\left(\frac{1}{\left|y_{\Psi Q}\right|}\right)^{2}\left(\frac{1}{\left|y_{\bar{L} D}\right|}\right)^{2}\left(\frac{M_{\Xi}}{4.9 \times 10^{10} \mathrm{GeV}}\right)^{4}, \\
& \tau_{p \rightarrow K^{+} \nu_{i}} \simeq\left(6.6 \times 10^{33} \mathrm{yrs}\right) \times\left(\frac{10^{-9}}{\frac{y_{D}^{\prime} v_{\mathrm{EW}}}{\sqrt{2}} / M_{\Psi}}\right)^{2}\left(\frac{1}{\left|y_{\Psi Q}\right|}\right)^{2}\left(\frac{1}{\left|y_{\bar{L} D}\right|}\right)^{2}\left(\frac{M_{\Xi}}{8.5 \times 10^{10} \mathrm{GeV}}\right)^{4},
\end{aligned}
$$

where $\left(\frac{y_{D}^{\prime} v_{\mathrm{EW}}}{\sqrt{2}} / M_{\Psi}\right)$ characterizes the mixing between the SM and KSVZ quark. Comparing the experimental bound of these two proton decay mode, $p \rightarrow K^{+} \bar{\nu}$ mode gives about an order of magnitude stronger bound than that on $p \rightarrow \pi^{+} \bar{\nu}$ mode. Thus, $p \rightarrow K^{+} \bar{\nu}$ mode is the most promising mode to explore this model.

The impact of the Higgs field insertion of the operators appears in the mixing between the SM and KSVZ quark, which is strongly suppressed by $v_{\mathrm{EW}} / M_{\Psi}$ Note that $v_{\mathrm{EW}}$ cannot be taken to be very large unlike the model discussed in the previous subsection. Even though the proton decay search has potential to probe high energy scale far beyond the collider reach if we assume the order one coupling constants.

\section{More axion models}

At $d=7$, there is a dressed operator $L L H H|H|^{2}$ with $\Delta \mathbf{L}=2$. At $d=8$, we have $\Delta \mathbf{B}=\Delta \mathbf{L}=1$ operators [15]
$\hat{\mathcal{O}}_{8}=\left\{u_{R} u_{R} Q L H^{c} H^{c}, d_{R} d_{R} Q L H H, d_{R} Q Q e_{R} H H, \hat{\mathcal{O}}_{6}|H|^{2}\right\}$,

where $\hat{\mathcal{O}}_{6}$ expresses dimension-six $\mathbf{B}+\mathbf{L}$ violating operators. These operators can also be used to construct an axion model. Since the model is controlled by the same symmetry, $\mathcal{O}_{7}=S \hat{\mathcal{O}}_{6}$ is allowed simultaneously with $\mathcal{O}_{9}=S \hat{\mathcal{O}}_{8}$. The effects of $\mathcal{O}_{7}$ are dominated in the low energy phenomena such as nucleon decays.

We can continue the same discussions with $d>8$ operators which contain lepton and baryon number violations. There are many variations of operators, which are characterized by $\Delta \mathbf{B}$ and $\Delta \mathbf{L}$ (see Fig. 1 of [15]). These operators can also be used to construct a model of axions.

A model for $n-\bar{n}$ oscillation: At $d=9$, there are purely baryon number violating six-quark operators with $\Delta \mathbf{B}=$ $2(\Delta \mathbf{L}=0)$. Therefore, the model predicts Sakharaxion without considering the lepton number violation. We here 
construct such a concrete model as an example. In order to build a model with minimal particle content, a color adjoint (Majorana) fermion $\Psi_{8}$ is introduced instead of the ordinary KSVZ quarks $\Psi$. Then, KSVZ Yukawa interaction is replaced by

$$
\mathcal{L}_{\Psi_{8}}=-\frac{1}{2} y_{8} S^{*} \overline{\left(\Psi_{8 L}^{A}\right)^{C}} \Psi_{8 L}^{A}+\text { H.c. }
$$

where $A=(1,2, \ldots, 8)$. A model with this Yukawa interaction is known as the gluino-axion model [46], where the quantum charges of $\Psi_{8}$ are the same as gluino in the supersymmetric theories. In addition, a QCD color antifundamental weak singlet diquark $\zeta$ with $Y=+1 / 3$ is introduced. The baryon number symmetry is assumed as the PQ symmetry with $\mathbf{B}\left(\Psi_{8}\right)=+1, \mathbf{B}(\zeta)=+2 / 3$ and $\mathbf{B}(S)=+2$. The relevant Lagrangian is given by

$$
\mathcal{L}=-y_{\zeta}^{i j} \epsilon_{a b c}\left(\zeta_{a}\right)^{*} \overline{\left(u_{i R}^{b}\right)^{C}} d_{j R}^{c}-y_{8 D}^{i} \zeta_{a}\left(T^{A}\right)^{a}{ }_{b} \Psi_{8 L}^{\bar{A}} d_{i R}^{b}+\text { H.c. }
$$

where $\left(T^{A}\right)^{a}{ }_{b}$ is the generator of $S U(3)_{C}$. This model predicts $\Delta \mathbf{B}=2$ process such as $n-\bar{n}$ oscillation as shown in Fig. 5. The low energy effective Lagrangian for the $n-\bar{n}$ oscillation is calculated as

$$
\mathcal{L}_{\text {eff }}^{\Delta \mathbf{B}=2}=C_{U U D D D D}^{i j k l m n} T_{[a b][c d]\{e f\}}^{A A S} \overline{\left(u_{i R}^{a}\right)^{C}} d_{j R}^{b} \overline{\left(u_{k R}^{c}\right)^{C}} d_{l R}^{d} \overline{\left(d_{m R}^{e}\right)^{C}} d_{n R}^{f}+\text { H.c. }
$$

with

$$
C_{U U D D D D}^{i j k l m n}=\frac{y_{\zeta}^{i j} y_{\zeta}^{k l} y_{8 D}^{m} y_{8 D}^{n}}{12 M_{\zeta}^{4} M_{8}}, \quad T_{[a b][c d]\{e f\}}^{A A S}=\epsilon_{a b e} \epsilon_{c d f}+\epsilon_{a b f} \epsilon_{c d e},
$$

where $M_{\zeta}$ and $M_{8}$ denote the masses of $\zeta$ and $\Psi_{8}$. Using the result of the neutron-antineutron matrix element [47], the $n-\bar{n}$ oscillation rate is estimated as

$$
\Gamma_{n \bar{n}}=\frac{10^{-9} \mathrm{~s}^{-1}}{(700 \mathrm{TeV})^{-5}}\left|(-4.2) \times \frac{1}{4} C_{U U D D D D}^{111111}\right|
$$

Replacing the Wilson coefficient by the model parameters, we found

$$
\tau_{n \bar{n}}=\Gamma_{n \bar{n}}{ }^{-1}=\left(7 \times 10^{8} \mathrm{~s}\right) \times\left(\frac{M_{\zeta}}{400 \mathrm{TeV}}\right)^{4}\left(\frac{M_{8}}{400 \mathrm{TeV}}\right)\left(\frac{1}{\left|y_{\zeta}^{11}\right|}\right)^{2}\left(\frac{1}{\left|y_{8 d}^{1}\right|}\right)^{2} .
$$

The current lower limit on neutron-antineutron oscillation is $\tau_{n \bar{n}}>4.7 \times 10^{8} \mathrm{~s}$, which is given by Super-Kamiokande [48]. Assuming the order one Yukawa coupling constants and the common new particle masses, the mass is constrained to be larger than $400 \mathrm{TeV}$. This process explores much higher

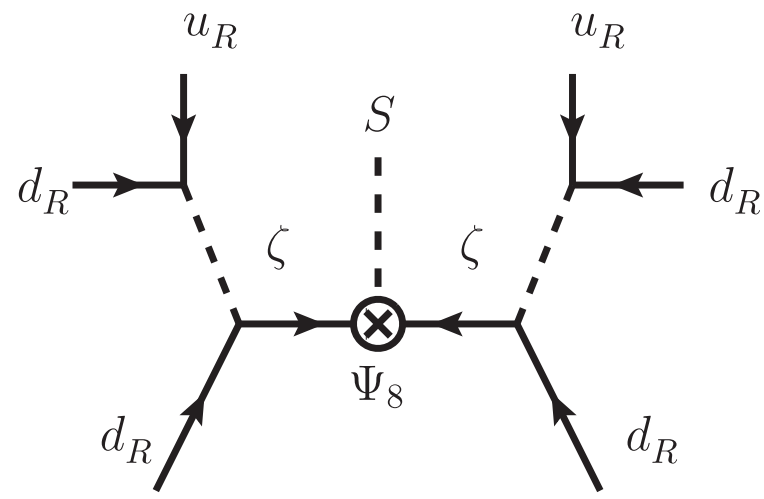

FIG. 5. Diagrams for $n-\bar{n}$ oscillation in a UV complete model. new physics scale than that of LHC direct searches for new colored particles. Comparably strong bounds of $10^{2-4} \mathrm{TeV}$ [49] may be obtained from neutral meson mixing, if the diquark $\zeta$ generates tree-level four fermion interactions with

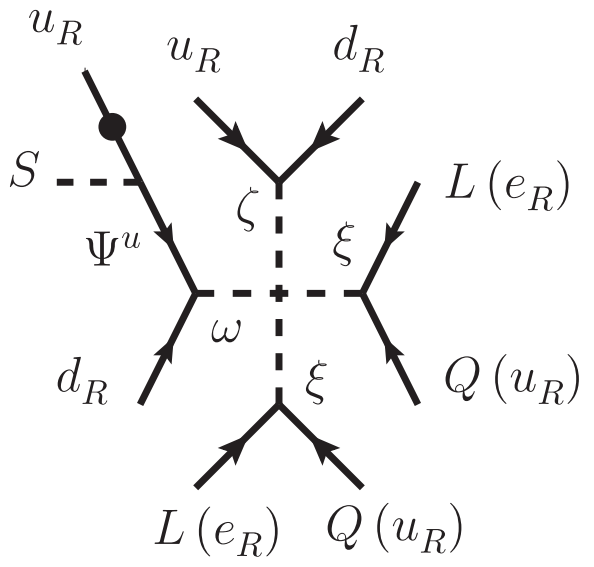


heavy quarks $s, c$ and $b$. If the diquark is assumed to interact only with the first generation quarks, we can avoid these flavor constraints since severe bounds come from $K, D$, and $B$ meson data. ${ }^{4}$ We also note that the model only predicts baryon number violations by two units, and thus no ordinary nucleon decay with $\Delta \mathbf{B}=1$ is induced.
A model for dinucleon decay: Another interesting baryon number violating processes are dinucleon decays, which are induced at $d=12$. Together with the QCD fundamental KSVZ quark $\Psi^{U}$ with $Y=+2 / 3$, one may introduce a leptoquark $\xi$, a diquark $\zeta$ and a tetraquark $\omega$ with $Y=-1 / 3$ and $\mathbf{L}(\omega)=+2$. The Lagrangian relevant to the dinucleon decay is given by

$$
\begin{aligned}
\mathcal{L}= & -y_{\Psi} S \Psi_{L}^{\bar{U} a} \Psi_{R}^{U a}-\mu_{U}^{i} \Psi_{L}^{\bar{U} a} u_{i R}^{a}-\left[y_{Q L}^{i j} \overline{\left(Q_{i}^{a}\right)^{C}}\left(i \sigma_{2}\right) L_{j}+y_{U E}^{i j} \overline{\left(u_{i R}^{a}\right)^{C}} e_{j R}\right]\left(\xi^{a}\right)^{*} \\
& -\epsilon_{a b c}\left[y_{Q Q}^{i j} \overline{\left(Q_{i}^{b}\right)^{C}}\left(i \sigma_{2}\right) Q_{j}^{c}+y_{U D}^{i j} \overline{\left(u_{i R}^{b}\right)^{C}} d_{j R}^{c}\right]\left(\zeta_{a}\right)^{*}-y_{\Psi D}^{i} \epsilon_{a b c} \overline{\overline{\left.\Psi_{R}^{U a}\right)^{C}}} d_{i R}^{b} \omega^{c} \\
& -\lambda^{\prime} \xi^{a} \xi^{b} \zeta_{a}\left(\omega^{b}\right)^{*}+\text { H.c. }
\end{aligned}
$$

We note that $\mathbf{B}\left(\Psi_{R}^{u}\right)=-5 / 3$. The dinucleon decay is generated by the diagram shown in Fig. 6 . The low energy effective Lagrangian for dinucleon decays is written as

$$
\mathcal{L}_{\text {eff }}^{\Delta(\mathbf{B}+\mathbf{L})=4}=C_{\text {UUUUDDEE }} T_{[a b][c d]\{e f\}}^{A A S} \overline{\left(u_{R}^{a}\right)^{C}} d_{R}^{b} \overline{\left(u_{R}^{c}\right)^{C}} d_{R}^{d} \overline{\left(u_{R}^{e}\right)^{C}} u_{R}^{f} \overline{e_{R}^{C}} e_{R}+\text { H.c. }
$$

with

$$
C_{U U U U D D E E}=-\frac{\lambda^{\prime} \mu_{U}^{1} y_{\Psi D}^{1} y_{U D}^{11}\left(y_{U E}^{11}\right)^{2}}{4 M_{\omega}^{2} M_{\zeta}^{2} M_{\xi}^{4} M_{\Psi}},
$$

where $M_{\xi}, M_{\zeta}$, and $M_{\omega}$ are masses of leptoquark, diquark, and tetraquark, respectively. Let us evaluate the dinucleon decay rate with this effective interaction. Here, we set the parameters as $y_{Q Q}=y_{Q L}=0$ for simplicity. Following Ref. [51], the width of the dinucleon decay is

$$
\Gamma_{p p \rightarrow e^{+} e^{+}} \simeq \frac{(\mathrm{TeV})^{16}}{2 \times 10^{26} \mathrm{yrs}}\left(\frac{\rho_{N}}{0.25 \mathrm{fm}^{-3}}\right)\left(\frac{m_{N}}{0.939 \mathrm{GeV}}\right)^{2}\left(\frac{\Lambda_{\mathrm{QCD}}}{200 \mathrm{MeV}}\right)^{12}\left|C_{\text {UUUUDDEE }}\right|^{2}
$$

where $m_{N}=\left(m_{p}+m_{n}\right) / 2$ is the mass of nucleon, $\rho_{N}\left(\sim 0.25 \mathrm{fm}^{-3}\right)$ is the average nuclear matter density, and $\Lambda_{\mathrm{QCD}}$ is the QCD scale parameter. In the present model, we found

$$
\begin{aligned}
\tau_{p p \rightarrow e^{+} e^{+}}= & \Gamma_{p p \rightarrow e^{+} e^{+}}^{-1} \simeq\left(5 \times 10^{33} \mathrm{yrs}\right) \times\left(\frac{M_{\omega}}{2 \mathrm{TeV}}\right)^{4}\left(\frac{M_{\zeta}}{2 \mathrm{TeV}}\right)^{4}\left(\frac{M_{\xi}}{2 \mathrm{TeV}}\right)^{8} \\
& \times\left(\frac{0.2}{\mu_{U}^{1} / M_{\Psi}}\right)^{2}\left(\frac{1}{\left|\lambda^{\prime}\right|}\right)^{2}\left(\frac{1}{\left|y_{U D}^{11}\right|}\right)^{2}\left(\frac{1}{\left|y_{U E}^{11}\right|}\right)^{4}\left(\frac{1}{\left|y_{\Psi D}^{1}\right|}\right)^{2} .
\end{aligned}
$$

The lower limit of the lifetime of the $p p \rightarrow e^{+} e^{+}$dinucleon decay mode is $\tau_{p p \rightarrow e^{+} e^{+}}>4.2 \times 10^{33} \mathrm{yrs}$ [52]. Even if we take order one new coupling constants, the common mass scale of new particles is about $2 \mathrm{TeV}$. This scale is almost the same as the current LHC bound on the colored new particles. Thus, the forthcoming high luminosity running of the LHC can help to test this model through the direct production of new colored particles. In order to avoid the constraint from the low energy flavor data, a specific flavor

\footnotetext{
${ }^{4} \mathrm{~A}$ comprehensive study for the diquark flavor structure is found, for example, in Ref. [50].
}

structure of the Yukawa coupling might be required. For example, if we assume that new colored particles solely couple the first generation fermions. In this case, effects on flavor changing decays of $\mu, \tau$ and mesons, and neutral meson mixing are forbidden at leading order. We also comment that no $\Delta \mathbf{B}=1$ proton decay as well as no $n-\bar{n}$ oscillation are predicted in this model.

\section{SUMMARY AND DISCUSSIONS}

PQ symmetry is often introduced in order to solve strong $C P$ problem and is sometimes linked to other new physics 
scenarios such as lepton number violating neutrino masses. Possible connections between the PQ symmetry and the baryon number and lepton number symmetries have been studied in the extensions of KSVZ model. Since the KSVZ solves the strong $C P$ problem in purely new physics sector, i.e., new KSVZ quark and a complex scalar, the baryon number and lepton number of new particles are undermined. In order to fix these quantum numbers, we have used the baryon and lepton number violating higher dimensional operators in the SM. Combining these operators with the scalar in the KSVZ model, we have developed the method to determine the baryon and lepton number of the new scalar. As a result, variants of the KSVZ axion model, which predict characteristic baryon number violations, are constructed. If we combine the scalar with $d=6(7) \mathbf{B}+\mathbf{L}(\mathbf{B}-\mathbf{L})$ violating operator, the axion model can also be explored through the nucleon decay experiment. With $d=9$ operator, the $n-\bar{n}$ oscillation is generated in the axion model. Since the VEV of the scalar violates the baryon number only by two units, $\Delta \mathbf{B}=1$ nucleon decay is forbidden in this model. Similarly with $d=12$ operator, the $\Delta(\mathbf{B}+\mathbf{L})=4$ dinucleon decay is predicted while no other baryon number violation is generated from the lower dimension operators. The experimental search for the baryon number violation are expected to be upgrade in the near future [53-55], it might be interesting to consider a diversity of baryon number violations other than the grand unified theories.

\section{ACKNOWLEDGMENTS}

We would like to thank Ernest Ma for pointing out the key idea of this paper and for a careful reading of our manuscript. This work was supported by MEXT KAKENHI Grant No. JP18H05543.
[1] C. Abel et al. (nEDM Collaboration), Phys. Rev. Lett. 124, 081803 (2020).

[2] G. 't Hooft, Phys. Rev. Lett. 37, 8 (1976); Phys. Rev. D 14, 3432 (1976); 18, 2199(E) (1978).

[3] R. D. Peccei and H. R. Quinn, Phys. Rev. Lett. 38, 1440 (1977); Phys. Rev. D 16, 1791 (1977).

[4] S. Weinberg, Phys. Rev. Lett. 40, 223 (1978); F. Wilczek, Phys. Rev. Lett. 40, 279 (1978).

[5] J. E. Kim, Phys. Rev. Lett. 43, 103 (1979); M. A. Shifman, A. I. Vainshtein, and V. I. Zakharov, Nucl. Phys. B166, 493 (1980).

[6] A. R. Zhitnitsky, Yad. Fiz. 31, 497 (1980) [Sov. J. Nucl. Phys. 31, 260 (1980)]; M. Dine, W. Fischler, and M. Srednicki, Phys. Lett. 104B, 199 (1981).

[7] Y. Ema, K. Hamaguchi, T. Moroi, and K. Nakayama, J. High Energy Phys. 01 (2017) 096.

[8] L. Calibbi, F. Goertz, D. Redigolo, R. Ziegler, and J. Zupan, Phys. Rev. D 95, 095009 (2017).

[9] Y. Chikashige, R. N. Mohapatra, and R. D. Peccei, Phys. Lett. 98B, 265 (1981); G. B. Gelmini and M. Roncadelli, Phys. Lett. 99B, 411 (1981).

[10] M. Shin, Phys. Rev. Lett. 59, 2515 (1987); 60, 383(E) (1988).

[11] E. Ma, Phys. Lett. B 514, 330 (2001).

[12] K. Abe, Y. Hayato, T. Iida, K. Iyogi, J. Kameda, Y. Kishimoto, Y. Koshio, L. Marti, M. Miura and S. Moriyama et al., Nucl. Instrum. Methods Phys. Res., Sect. A 737, 253 (2014); Y. Fukuda et al. (Super-Kamiokande Collaboration), Nucl. Instrum. Methods Phys. Res., Sect. A 501, 418 (2003).

[13] S. Weinberg, Phys. Rev. Lett. 43, 1566 (1979).

[14] F. Wilczek and A. Zee, Phys. Rev. Lett. 43, 1571 (1979).

[15] J. Heeck and V. Takhistov, Phys. Rev. D 101, 015005 (2020).
[16] P. Minkowski, Phys. Lett. B 67, 421 (1977); T. Yanagida, in Proceedings of the Workshop on Unified Theory and Baryon Number of the Universe, edited by O. Sawada and A. Sugamoto (KEK, Tsukuba, 1979); M. Gell-Mann, P. Ramond, and R. Slansky, in Supergravity, edited by P. van Niewenhuizen and D. Freedman (North Holland, Amsterdam, 1979); S. L. Glashow, NATO Sci. Ser. B 61, 687 (1980); R. N. Mohapatra and G. Senjanovic, Phys. Rev. Lett. 44, 912 (1980).

[17] P. Langacker, R. D. Peccei, and T. Yanagida, Mod. Phys. Lett. A 01, 541 (1986); J. D. Clarke and R. R. Volkas, Phys. Rev. D 93, 035001 (2016).

[18] J. Quevillon and C. Smith, Phys. Rev. D 102, 075031 (2020).

[19] P. Sikivie, Phys. Rev. Lett. 48, 1156 (1982).

[20] J. Schechter and J. W. F. Valle, Phys. Rev. D 22, 2227 (1980); M. Magg and C. Wetterich, Phys. Lett. B 94, 61 (1980); G. Lazarides, Q. Shafi, and C. Wetterich, Nucl. Phys. B181, 287 (1981).

[21] S. Bertolini, L. Di Luzio, H. Kolešová, and M. Malinský, Phys. Rev. D 91, 055014 (2015).

[22] Y. H. Ahn and E. J. Chun, Phys. Lett. B 752, 333 (2016).

[23] R. Foot, H. Lew, X. G. He, and G. C. Joshi, Z. Phys. C 44, 441 (1989).

[24] E. Ma, Phys. Rev. Lett. 81, 1171 (1998).

[25] Y. Cai, J. Herrero-García, M. A. Schmidt, A. Vicente, and R. R. Volkas, Front. Phys. 5, 63 (2017).

[26] B. Dasgupta, E. Ma, and K. Tsumura, Phys. Rev. D 89, 041702 (2014).

[27] E. Ma, T. Ohata, and K. Tsumura, Phys. Rev. D 96, 075039 (2017).

[28] A. Zee, Phys. Lett. B 93, 389 (1980); 95, 461(E) (1980). 
[29] S. Bertolini and A. Santamaria, Nucl. Phys. B357, 222 (1991); H. Arason, P. Ramond, and B. D. Wright, Phys. Rev. D 43, 2337 (1991).

[30] A. Zee, Nucl. Phys. B264, 99 (1986); K. S. Babu, Phys. Lett. B 203, 132 (1988).

[31] E. Ma, Phys. Rev. D 73, 077301 (2006).

[32] E. Ma, Phys. Rev. Lett. 115, 011801 (2015).

[33] S. Y. Ho, T. Toma, and K. Tsumura, Phys. Rev. D 94, 033007 (2016).

[34] L. M. Krauss and F. Wilczek, Phys. Rev. Lett. 62, 1221 (1989).

[35] Y. Aoki, T. Izubuchi, E. Shintani, and A. Soni, Phys. Rev. D 96, 014506 (2017).

[36] A. Takenaka et al. (Super-Kamiokande Collaboration), Phys. Rev. D 102, 112011 (2020).

[37] K. Abe et al. (Super-Kamiokande Collaboration), Phys. Rev. Lett. 113, 121802 (2014).

[38] K. Kobayashi et al. (Super-Kamiokande Collaboration), Phys. Rev. D 72, 052007 (2005).

[39] C. Regis et al. (Super-Kamiokande Collaboration), Phys. Rev. D 86, 012006 (2012).

[40] K. Abe et al. (Super-Kamiokande Collaboration), Phys. Rev. D 90, 072005 (2014).
[41] V. Takhistov (Super-Kamiokande Collaboration), arXiv: 1605.03235 .

[42] K. Abe et al. (Super-Kamiokande Collaboration), Phys. Rev. D 96, 012003 (2017).

[43] E. Ma, Mod. Phys. Lett. A 32, 1730007 (2017).

[44] G. G. Raffelt, Lect. Notes Phys. 741, 51 (2008).

[45] M. S. Turner, Phys. Rev. D 33, 889 (1986).

[46] D. A. Demir and E. Ma, Phys. Rev. D 62, 111901 (2000).

[47] E. Rinaldi, S. Syritsyn, M. L. Wagman, M. I. Buchoff, C. Schroeder, and J. Wasem, Phys. Rev. Lett. 122, 162001 (2019).

[48] K. Abe et al. (Super-Kamiokande Collaboration), Phys. Rev. D 103, 012008 (2021).

[49] G. Isidori, Adv. Ser. Dir. High Energy Phys. 26, 339 (2016).

[50] G. F. Giudice, B. Gripaios, and R. Sundrum, J. High Energy Phys. 08 (2011) 055.

[51] X. G. He and X. D. Ma, J. High Energy Phys. 06 (2021) 047.

[52] S. Sussman et al. (Super-Kamiokande Collaboration), arXiv:1811.12430.

[53] Z. Djurcic et al. (JUNO Collaboration), arXiv:1508.07166.

[54] R. Acciarri et al. (DUNE Collaboration), arXiv:1512.06148.

[55] K. Abe et al. (Hyper-Kamiokande Collaboration), arXiv: 1805.04163. 